\title{
Simulation and Track Reconstruction Techniques for the J-PARC muon g-2 experiment
}

\author{
Paschalis Tsilias ${ }^{1, a}$ and Dr. MyeongJae Lee ${ }^{2}$ \\ on behalf of J-PARC $\mathrm{g}$-2/EDM collaboration \\ ${ }^{1}$ Deparment of Physics, Aristotle University of Thessaloniki, Greece \\ ${ }^{2}$ Center for Axion and Precision Physics Research, Institute for Basic Science, KAIST, South Korea
}

\begin{abstract}
The Muon g-2/EDM proposed experiment at J-PARC is a promising and innovative attempt at the field of Precision Physics. The sensitivity goal of $0.1 \mathrm{ppm}$ will test the limits of our current understanding, and may probe for Beyond the Standard Model observations. This paper seeks out to investigate the computational techniques required by the experiment. The GEANT4 [1] framework was used to simulate the detector setup, according to the experiment's Conceptual Design Report (CDR) [2]. This allowed to observe the event hierarchy in different energies, generate signal hit data, and construct an event-selection algorithm. ROOT and GDML enabled us to use the geometry and parsed output data in a platform-independent way. Using techniques pertaining to Machine Learning and Image Feature extraction, such as the Canny Edge detection and the Hough Transform, we were able to construct a generic representation of 'track families' from each event category. Finally, the modular GENFIT2 [3] framework was used to implement the Kalman Filter [4] along with an Deterministic Annealing Filter (DAF) [5] and the Runge-Kutta stepper to reconstruct tracks from a few digitized, smeared singular event data.
\end{abstract}

\section{Introduction}

The Standard Model (SM) has been one of the crowning achievements of human intellect. For the last 50 years it has led to the discovery of new particles, with the most recent being the Higgs boson, as well as helped give rise to the most precise theory ever, in the form of QED.

One of the most precisely measured quantities in particle physics, is the muon anomalous magnetic moment factor, $\alpha_{\mu}$, which has served as a concrete testing ground for SM predictions. The 2006 muon g-2 experiment in Brookhaven [6] reached unprecedented precision of $0.54 \mathrm{ppm}$, and constrained $d_{\mu}$ to an upper limit o $1.9 \times 10^{-19} e \cdot \mathrm{cm}$. When their results were published, a persistent discrepancy of $\sim 3.4 \sigma$ was observed for $\alpha_{\mu}$, while the $d_{\mu}$ limit requires improvement to test against the SM prediction, thus marking the largest measured deviation for the Standard Model. This statistically significant deviation, while not yet definitive, suggests that there may be effects on the muon's anomalous magnetic moment factor, that cannot be explained in the SM framework. The potential discovery of concrete evidence of new physics, beyond the Standard Model provides motivation of further studies and improved measurement of the $\alpha_{\mu}$.

\footnotetext{
ae-mail: ptsilias@physics.auth.gr
} 
Two major attempts are currently in progress, one in Fermilab, where an upgraded version of their E821 experiment is expected to begin gathering data on 2017, and one in J-PARC, where an original, new approach, is considered for the experiment, and is still a few years away. Both experiments have set a sensitivity goal of $\Delta \alpha_{\mu}=0.1 \mathrm{ppm}$.

\section{The J-PARC muon g-2 experiment}

The Conceptual Design Report (CDR) for the J-PARC muon g-2 experiment was published in December 2011. The first submitted proposal to the J-PARC committee was back in December 2009, and has undergone significant R\&D efforts and feasibility reports. In the following paragraph, the authors frequently quotes the CDR directly, to preserve the original ideas clearly.

The CDR, where the present paper is based on, contains the 'baseline design for the muon g2/EDM experiment proposed for the J-PARC muon facility at MLF'. The sensitivity goal was set at $0.1 \mathrm{ppm}$, being able to probe for new physics, along with the upgraded Fermilab experiment.

The experimental idea, summarized in a sentence or two, is 'storing $\mu^{+}$in MRI-type solenoid magnet, and measure polarized $\mu^{+}$decays to positrons'. Higher energy $e^{+}$are emitted with the muon spin direction aligned with along its trajectory in the storage ring. The muon spin precesses from the forward to the backward direction, along its momentum vector. This precession effect, due to an advance of the spin precession compared to the muon momentum in a magnetic field, is due to the difference $g-2$.

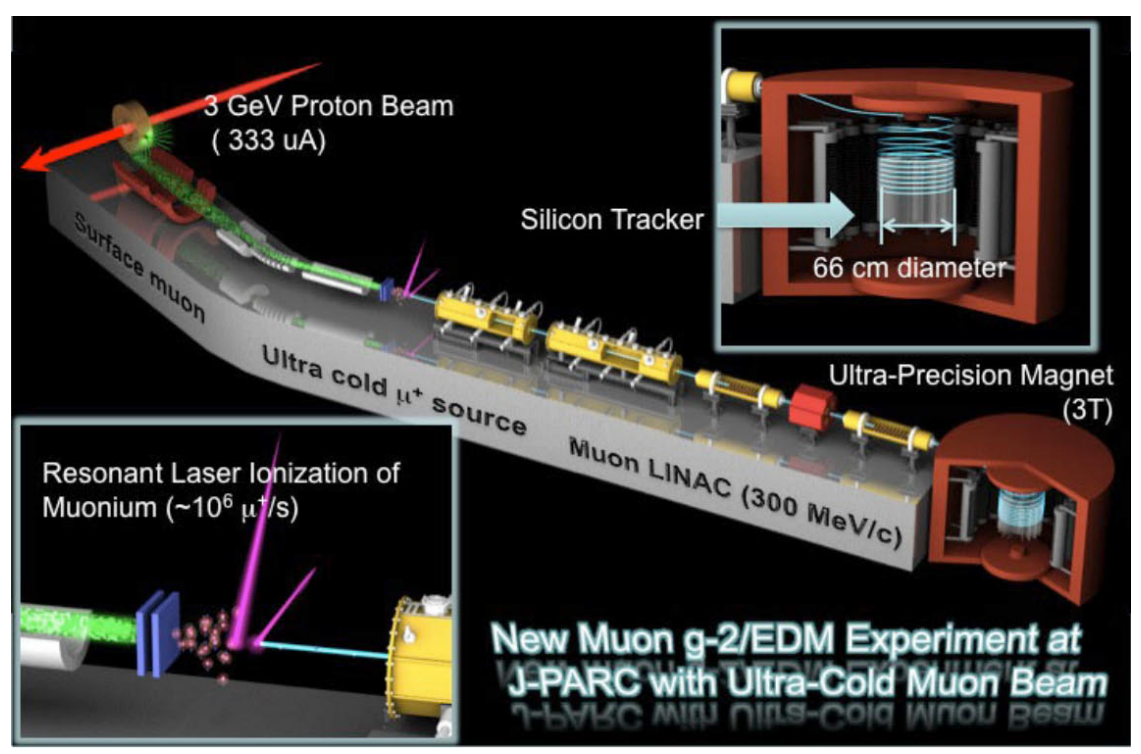

Figure 1. Generic representation of the experiment facility. [2]

A detector setup inside of the muon storage orbit will track the decay $e^{+}$versus the time in store, as the muon spin precesses. The anomaly $\mathrm{g}-2$ is the advance of the muon spin from its momentum vector, divided by the storage magnet field strength. As such, the g- 2 experiment requires precision measurements of the higher energy $e^{+}$with respect to time and the magnetic field's value.

The EDM measurement will be achieved using the same setup, as both dipole measurements can be done at the same time. This requires measuring the precession frequency as a vector, with one 
component parallel to the magnetic field, and one component orthogonal to the momentum vector and the magnetic field.

This experimental setup attempts to use a different approach to previous muon g-2 experiments, using an order of magnitude lower energy and an order of magnitude smaller diameter of the storage ring. Such an approach motivates the use of an ultra-cold muon source, that requires only weak focusing to maintain the beam size in the storage ring, leading to the choice of a 3T MRI-type solenoid for the storage phase, and a momentum of $300 \mathrm{MeV} / \mathrm{c}$.

The lower energy will allow to frequently reverse the muon spins, to cancel systematic errors that are difficult to deal with, such as the pile-up effect, something that has not been used in previous attempts.

\section{Simulation of Events}

The simulation was implemented in the definitive GEANT4 framework, which has been widely adopted in both academia and the industry. It includes the spatial motion of muons, their decay, the positron propagation code, tracking of secondary particles, as well as the positron interaction with the detector material. In some aspects, the 'ideal case' approach was used, in order to investigate the event structure of the experiment and allow to get a general understanding of the underlying physics concepts

We focus the late phase of the experiment, from the point of particle injection in the solenoid storage area. The stored $\mu^{+}$travel in circular trajectories, under a uniform magnetic field, around the detector setup.

Once the muon decays according to

$$
\mu^{+} \longrightarrow e^{+}+v_{e}+\overline{v_{\mu}}
$$

the produced positron follows an inwards helix-elliptic trajectory, where it deposits energy in the silicon vanes, and generates hit positions.

The detector is composed of 48 silicon vanes, radially located around a central cylindrical tungsten volume, whose goal in the complete setup is to absorb positrons with middle and low energy, to avoid many turns of positrons passing through the center volume. Dr. MyeongJae Lee, proposed that we remove this 'absorber' in order to better monitor the event hierarchy and material energy depositing effects, so that we get a more complete understanding of the event-selection algorithm.

There are 48 identical 'vanes' with $(220 \times 400 \times 0.03 \mathrm{~mm})$ size, perpendicular to the $\mathrm{X}-\mathrm{Y}$ plane, evenly distributed around the World volume center (Point $0,0,0$ ) rotated in the Z-axis by $7.5^{\circ}$ each.

Next, we simulate a uniform magnetic field, in the Z-axis direction, with a value of -3 Tesla. A general description of EM field that can be used in a more realistic simulation, was also developed, but for the time being, we oped to use the ideal case. For example a more detailed approach would include the electric field from the detector and readout electronics, but it shouldn't be large enough to provide any noise in the measurement process $(10 \mathrm{mV} / \mathrm{cm}$ for $0.1 \mathrm{ppm}$ systematic uncertainty).

The experimental setup is designed to be sensitive to positrons with energies between $\sim 50$ and $\sim 280 \mathrm{MeV}$. Positrons with higher energies have tracks similar to the muons, outside of the vanes setup, while those with lower energies, the curl radius is small, and introduces noise effects.

As for the particle transport code, the implemented Stepping utilizes the classical fourth-order Runge-Kutta stepper, that provides a good balance between precision of the simulated step and CPU time. The 'maximum step' that is allowed by the simulation is well below the vane width. Our simpler approach does not simulate the injection phase with the kicker field, but rather relies on already-injected particles. The particle source, located 330mm along the $\mathrm{X}$ axis, emits muons with an 


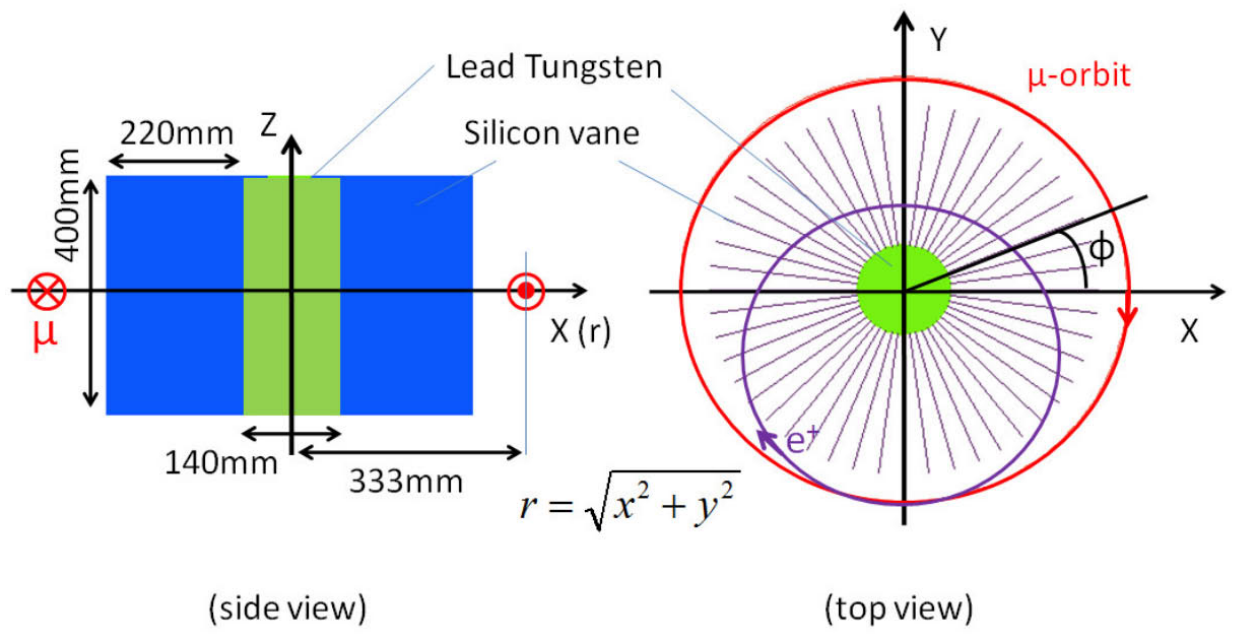

Figure 2. Scheme diagram of the detector setup - Axes definition.

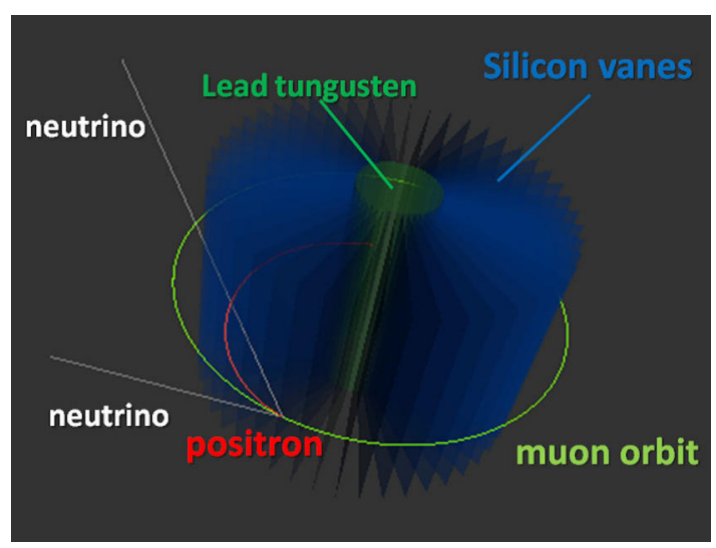

Figure 3. 3D render of the detector, and mock trajectories.

energy of $300 \mathrm{MeV}$, while the momentum direction is represented by a $(0,1,0)$ vector. As the muon lifetime at this energy scale is deemed quite long, GEANT4 mistakes the circular motion as a stable setup. For this reason, the muon's lifetime had to be artificially lowered to $0.5 \mathrm{~ns}$ so that there were decays around its trajectory.

The final output data from the simulation is the energy of the produced secondary particles, as well as the hit coordinates in the silicon vanes. For scoring purposes, Sensitive Detector modules were implemented in the Silicon volumes. This module generates a Hit Collection for each muon. Whenever a 'step' during a secondary particle propagation deposits energy on a Silicon Volume a new Hit is recorded and visualized. For more precise measurements we use the information from both GetPreStepPoint() and GetPostStepPoint(), as the scattering angle alters the entry and exit hit coordinates. A feature of the project was the detachment the Geant 4 framework from the Data Analysis 

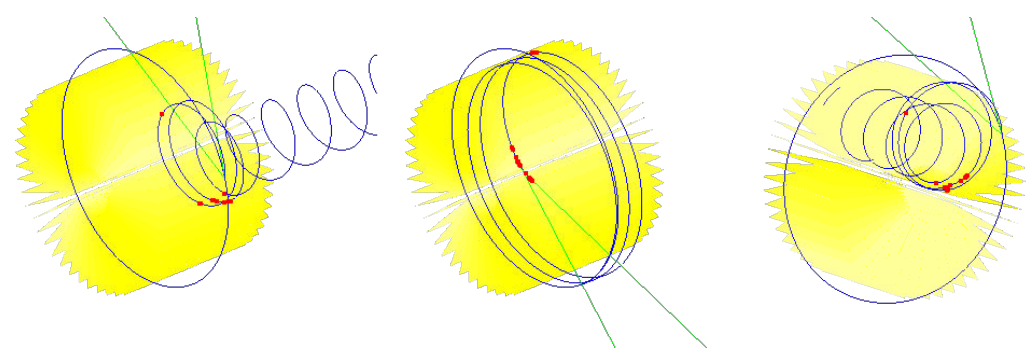

Figure 4. Generic representation of the experiment facility.

and Track Reconstruction procedure. For this reason the GMDL schema was used, to describe the geometry in a platform-independent format. GDML files can be used in ROOT, CAD applications, as well as the chosen GENFIT framework.

\section{Event Selection}

As per the Conceptual Design Report, the detected events can be divided in 8 categories, that are greatly dependent on the initial positron energy. A large amount of primary events $(\sim 40 \%)$ do not generate hits in the silicon tracker, either because they have lower energy than the necessary threshold, due to the decay direction, or due to higher energy, where their trajectory is similar to that of the muons. The general hierarchy that is observed at the CDR is found here as well.

To categorize the events, the $\mathrm{X}-\mathrm{Y}$ and $\phi-Z$ plane hit scatter plots were used. Other criteria include the radius of the curved trajectory, the number of 'turns', the presence of secondary electrons, as well as the hit angle of the positrons. This separation is critical for the analysis project, because each category needs has a different ratio of usable/background events. Following the CDR, and using the aforementioned criteria, the events were separated as

1 Clear Event - The simplest case, where the only algorithm needed is finding the line trace in the $\phi Z$ plane. This constitutes the clearest signal event.

2 Clear Event w/ secondary electrons - In addition to the clear trace, there is a secondary trace stemming from hitpoints by electron hits ( $\delta$ ray EM shower) that are considered noise, and must be rejected before the data analysis.

3 Small Curls - There are positron curls, with a small enough radius to not reach the center region. The $\phi Z$ trace is comprised by parallel lines. Due to the low energy, most events in this category are considered background, and removed.

3b Small Curls w/ secondary electrons - The events have both small curls, and secondary electrons, like (2), and are considered background.

$4 \quad$ Middle curls - The positron curls have a larger radius, passing through the vanes without hits in the center region. Most of these events have middle energy, and almost all of them are considered useful signal events. They leave multiple-lines trace in the $\phi Z$ plane. An algorithm recognizing the initial hit point, near the $Z=0$ can help future analysis. 
4b Middle curls w/ secondary electrons. This event has both the middle curl characteristic, as well as the smearing from secondary electrons. In order for these events to be useful, an algorithm must be developed to separate the signal track from th noise.

5 Large curls - The positron tracks passes around the center region with a bigger radius. Similar to category (4) there are line-like traces in the $\phi Z$ plane, and due to the larger positron energy, almost all events are considered signal tracks.

5b Large curls w/ secondary electrons - The events have a large curl, with noise from secondary electron, that should be reduced or removed.

6 Only multiple scattering - The positron is scattered with a large angle, depositing energy in the silicon vane. The $\phi Z$ plane is a characteristic broken line. In order for these events to be used, the algorithm must be able to recognize this difference.

6b Multiple scattering with many curls or secondary electrons

7 Others - A positron of larger energy can provide hit points from categories $(2)+(3)+(4)$, and is the most complicated to recognize. The algorithm to separate these events can also use the time-parameter, as positrons with such large energies tend to drift in the silicon vanes for longer times.

No hit These null events do not provide any silicon vane hits. That can be attributed to very low $(<50 \mathrm{MeV})$, very large $(E>280 \mathrm{MeV})$ positron energy, or outwards positron trajectory.

Even though limitations are imposed by statistics, we can verify the general observation and confirm that, at low energy region, the largest events were in category (3), at middle energy range, categories (1)and (2) are dominant, the events in categories (4) - (7) increase with the energy.
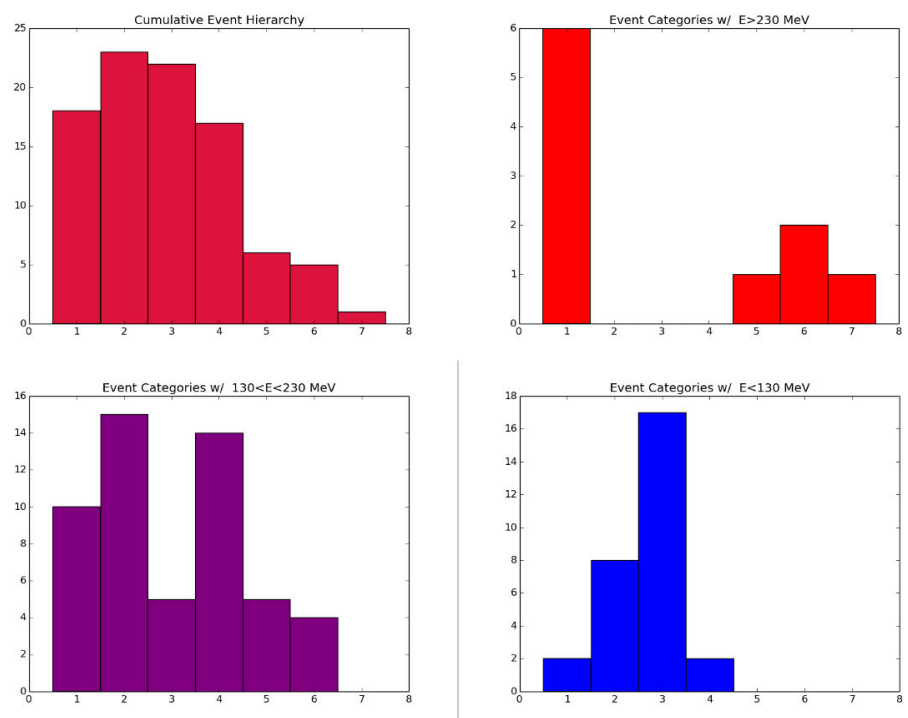

Figure 5. Observed Hierarchy of 100 Simulated Events. 

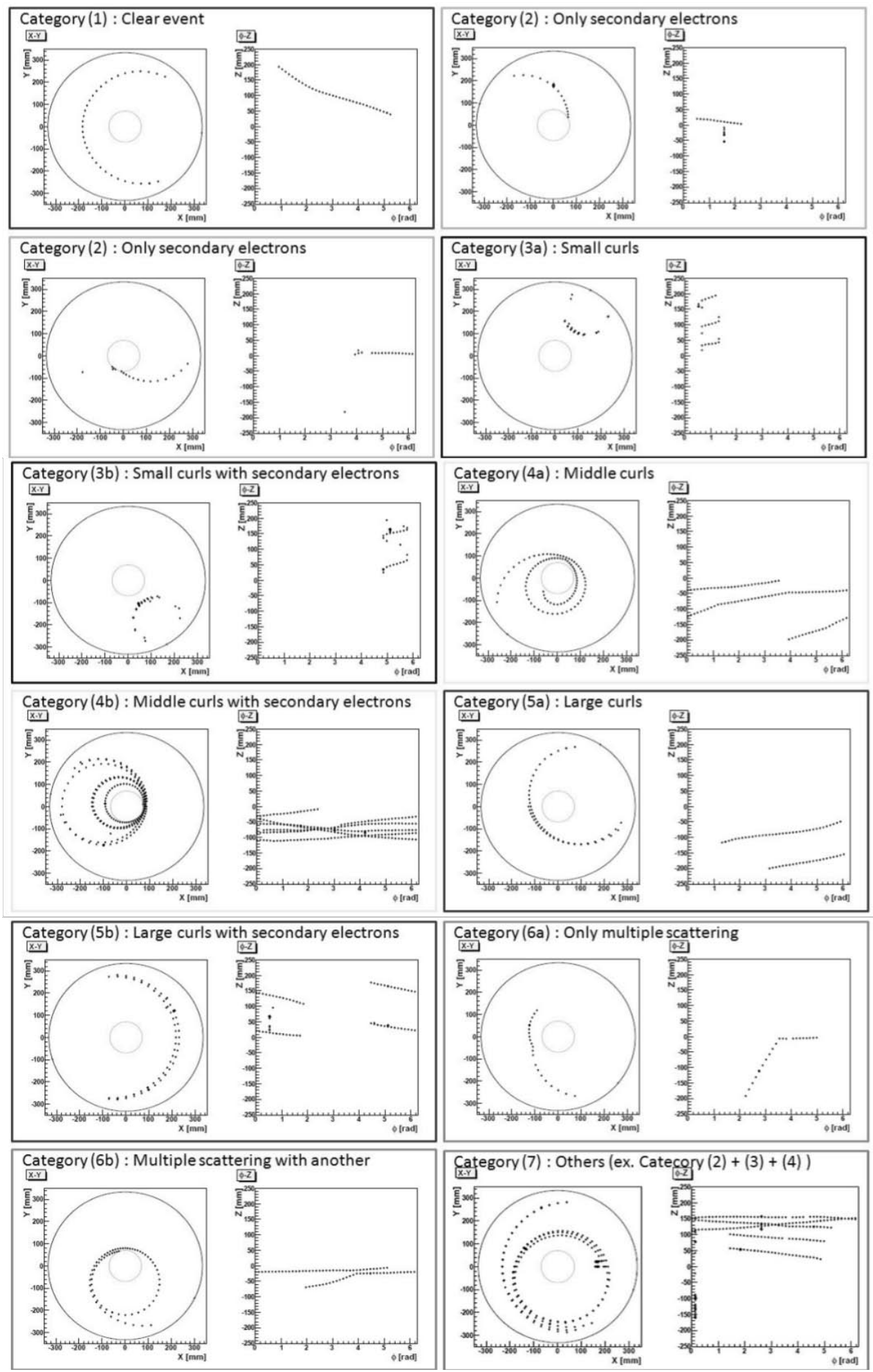

Figure 6. Overview of $\mathrm{X}-\mathrm{Y}$ and $\phi \mathrm{Z}$ traces from the CDR. 


\section{Track Reconstruction}

As stated, the precise measurement and reconstruction of the decaying positron track is the most critical part of this experiment. The GENFIT2 is a very promising, and quite new framework, developed at TUM, that is slowly gaining traction in experiments such as Belle II, PANDA, GEM-TPC, FOPI, and one of the reasons it was selected, was to investigate its usefulness. As we already mentioned,the framework's main attraction is its modular structure. This allows to decouple the algebra of the regression algorithm from the hit representation and track representation.

\subsection{Visualization and Geometry}

For visualization purposes, the framework uses the gEVE module from ROOT. The geometry was imported in the GENFIT2 framework using the GDML schema, and a subsequent conversion to a .ROOT file.

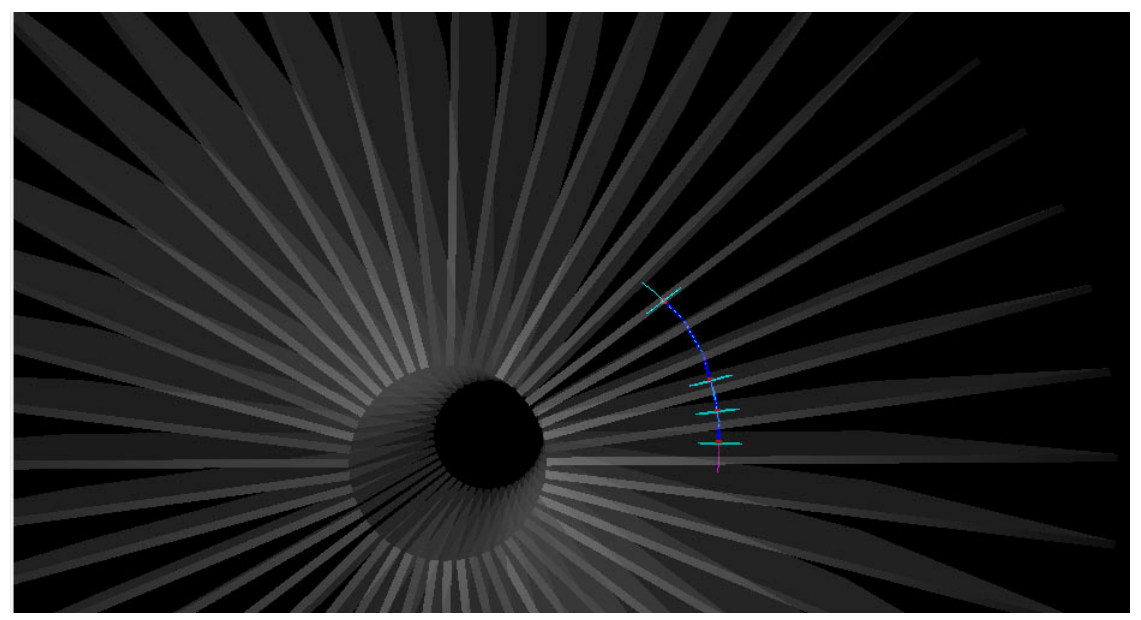

Figure 7. Early implementation of geometry definitions and detector planes on GENFIT2.

\subsection{Hit Representation}

To be able to define hit points on our detector, one measurement plane per vane was assigned. GENFIT2 constructs measurement planes, by using a point as the origin of the plane, as well as two orthogonal vectors. We opted to use the origin of the axes as the starting point, and the two orthogonal vectors being across the $\mathrm{Z}$-axis as well as parallel to the vane plane $(\cos \theta-\sin \theta)$. This way, the three parameters that GENFIT requires to assign a hit point, is the Vane detector angle (or number), $\mathrm{XY}$ plane radius, and the $\mathrm{Z}$-value of the hit point.

\subsection{Regression Algorithm}

Kalman Filters [4] are widely used in High Energy Physics studies and track fitting. The algorithm is based on an iterative least square fit of the track state. The iteration uses a prediction step, to 
extrapolate the state vector to the next layer, as well as an update step to the best-fit predicted state. Kalman Filters can work with the classical fourth-order Runge Kutta stepper, and just the physical properties of the detector volume, propagating the particle from one surface to another, adding up scattering and precise energy loss effects. In this paper, mainly due to the time limit, we focused

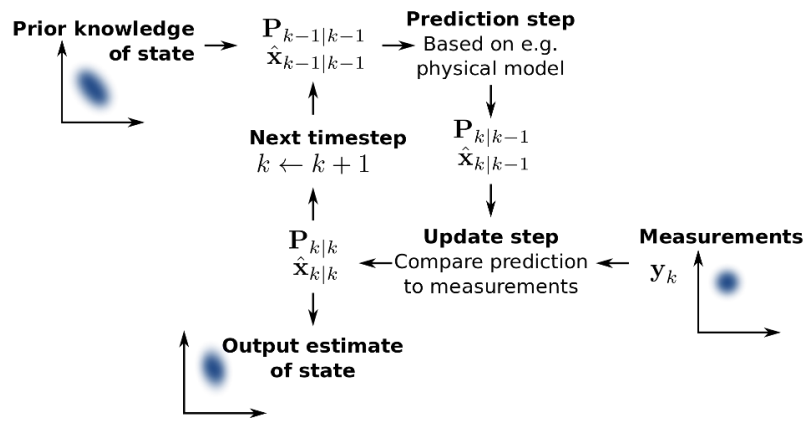

Figure 8. Schematic of the basic Kalman filter concept, from Wikipedia.

on reconstructing singular positron tracks. When we have proper understanding of the underlying physical processes, and the tools and methods used have matured it may be possible to scale them into using for multi-tracks analysis.

\subsection{Pattern Recognition}

In such cases, where we work with sparse hit points data, for example in non-consecutive vanes, or when we try to analyze very complicated patterns from multi-muons tracks, the Kalman filter might be insufficient. A more complicated X-Y and $\phi Z$ plane trace, that contains multiple muons track, require some pattern recognition algorithms to be able to make a distinction between background events, and useful signal data. Note that, the average number of muon decays in the first 5 ns provides about 30 events. Figure 9, from the experiment's CDR, shows an example of the complicated state, where the aforementioned pattern recognition algorithm's use is justified.

For this reason, and working with these pattern recognition techniques, the end goal is to provide a Track Model, as a Reference State (the 'Seed Fit'). This way, the Kalman filter's prediction step has a starting value to extrapolate from. This 'Seed Fit' must be derived from a careful event selection and analysis, using the categorizing algorithm we began to describe in the previous section.

As we saw, the singular positron tracks in the silicon detector setup left line-like traces in the $\phi Z$ plane. For this reason,the Hough Transform can be an invaluable tool. Also, the individual significance of each hit coordinate can be calibrated with the Canny Edge detection algorithm. This way, tightly grouped points can be recognized and either removed, or assigned a lower weight value.

The mathematical concept of the Hough Transform is not that complicated, and it is possible to write the necessary code from scratch. But in order to be more flexible and secure about our results, we opt to use an already existing, tested, implementation that has been used in the industry with success. As such the OpenCV [7] (Open Source Computer Vision) framework was chosen, which is a set of libraries mainly aimed at real-time computer vision, originally developed by Intel.

Two examples uses of the Canny Edge detection, along with the Hough Transform, for two example run data sets are presented. 

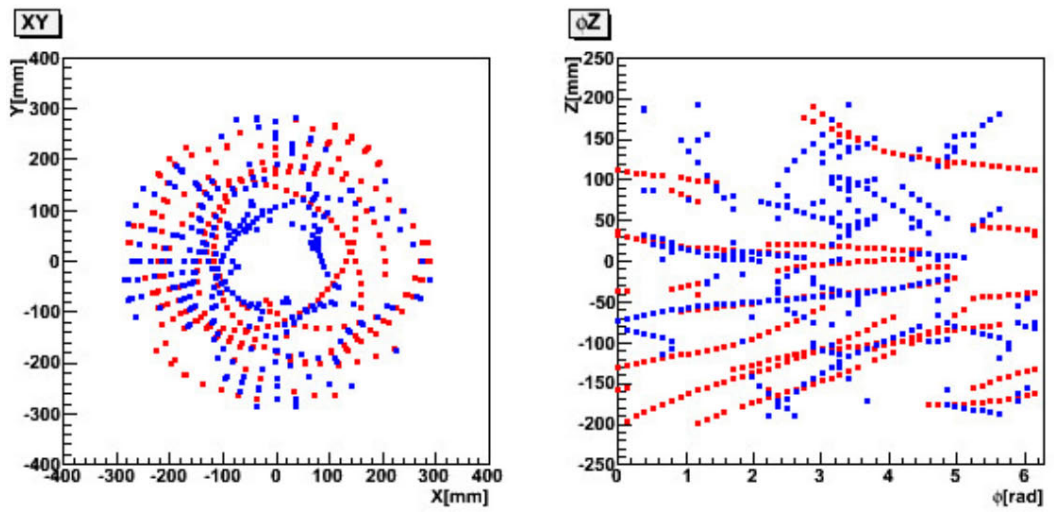

Figure 9. XY-plane and $\phi Z$-plane traces for multi-muon tracks, from the CDR. Red points represent signal tracks where $E_{\text {initial }}>200 \mathrm{MeV}$. Blue ones show background tracks.

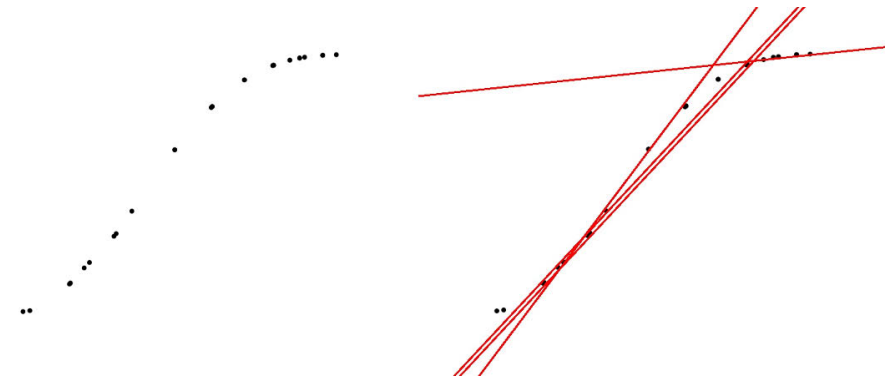

Figure 10. Example of Hough Transformation on $\phi Z$ plane trace.

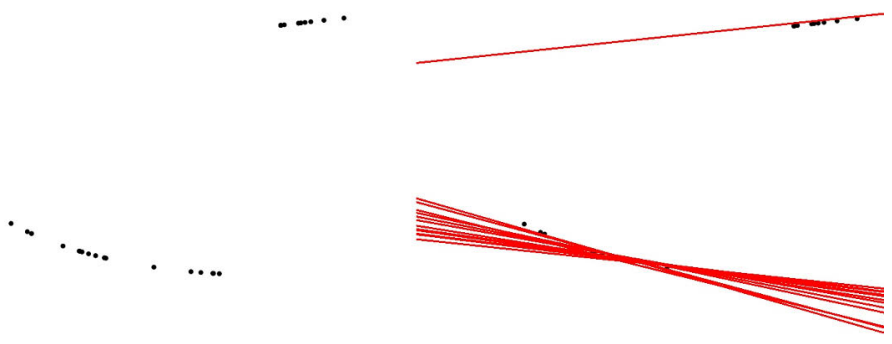

Figure 11. Example of Hough Transformation on $\phi Z$ plane trace.

Unfortunately, the complicated state of some track events, led us to use a simple Kalman filter, without using the generic track representation, as the Reference state. For this reason, the initial idea was to work with the local track reconstructing, using bunches of three to five points, and having a basic quality control of this local approach. 


\subsection{Track Reconstruction}

Using the aforementioned Hit Representation, along with the classical fourth-order Runge Kutta stepper, the .ROOT file for our geometry, and with GenFit handling the Material Effects, it is possible to begin reconstructing tracks from the Geant 4 simulations.

In order to reproduce the digitization effect, as the silicon strips have a finite resolution, the detector hit points were smeared by inserting the planar detector resolution into the covariance matrix. To help the Kalman Filter to cope with the digitized data, we introduced the Deterministic Annealing Filter (DAF), that has been successfully used in the ATLAS experiment, at LHC.

Validating the precision of the material effect is a much harder job than we can allocate time to, even though it is one of the most crucial parts of the experiment. But we can simply check their presence and implementation by using dummy data, and switching Material Energy Loss effects On and Off.

Having established a stable workflow, it is possible to reconstruct any hitpoints dataset from the Si vanes, originating from the Geant4 simulation.

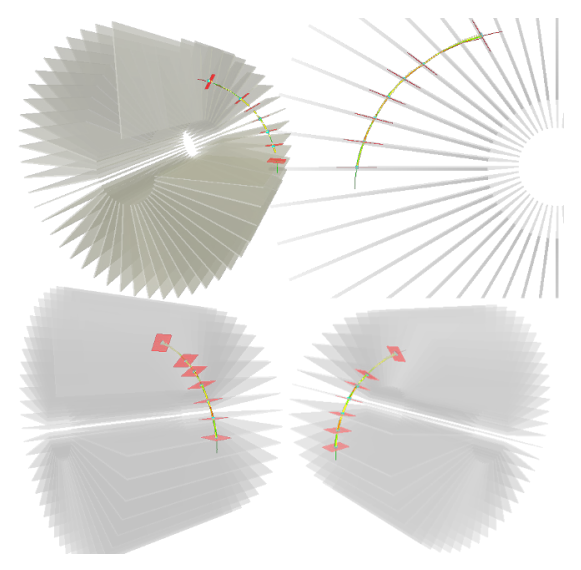

Figure 12. Track Reconstruction from Geant 4 event WITH Material Effects in place.

\section{Conclusions - Results}

This paper started as a project for the KAIST 2016 summer research program. The authors developed a complete proof-of-concept framework, creating original, exciting code in the process. Using Geant4, ROOT, and the GENFIT2 framework, the established workflow can successfully reconstruct simulated simple tracks for the muon $\mathrm{g}-2$ experiment.

More specifically, it allows us to

- Simulate the late phase of the experiment

- Focus on the precision factors of the simulation

- Analyze the observed even hierarchy and categorization according to the initial positron energy

- Describe a basic Event Selection Algorithm

- Implement the necessary algorithms for the Pattern Recognition 
- Develop a Hit Representation algorithm for our geometry and explore the material effects of the framework

- Represent Digitization effects

- Implement the Kalman Filter along with an Deterministic Annealing Filter (DAF) and the RungeKutta stepper

- Finally Reconstruct Simulated Tracks from a few digitized, smeared singular event data

\section{Discussion - Future goals}

While providing some first results, this project has the potential to go into greater lengths, that unfortunately, the initial timeframe did not allow us to explore. The current goal is to get a measurable quality factor between the Geant 4 simulated steps, and the Reconstructed Tracks, by using one every 500 or 1000 Geant 4 steps.

The biggest challenge would be trying to implement spin polarization in the GEANT4 code, something that people at KAIST are currently working on.

The next logical step be implementing the tungsten absorber, and trying to simulate a more realistic setup, taking into account various technical limitation and deficiencies. That includes a realistic EM field representation, a realistic construction of the silicon detector, that includes 'blind spots' at the electronics, as well as introducing the digitization representation into GEANT4.

A more detailed approach would try to validate the low energy positron-matter that are used in GEANT4, using the appropriate low energy packages for the physics list, as well as the validity of material effects used in GENFIT2

Finally, simulating the muon transport and injection phase, while more complicated, could provide better insight into the experiment's properties. Such attempts to implement a better beam simulation as well as the kicker injection field, is on the experiment's roadmap.

\section{References}

[1] The Geant4 collaboration, The Geant4 framework for Monte Carlo simulations - Documentation, (2016), https://geant4.web.cern.ch/geant4/

[2] The Muon g-2 collaboration, Conceptual Design Report for The Measurement of the Muon Anomalous Magnetic Moment g-2 and Electric Dipole Moment at J-PARC, (2012) https://g2sakura.kek.jp/public/doc/MCDR-submit.pdf

[3] C. Höppner, S. Neubert, et. al, A Novel Generic Framework for Track Fitting in Complex Detector Systems (2010), arXiv:0911.1008 [hep-ex]

[4] Mu2e Tracking Software Workshop” proceedings, (2012)

http://mu2e.fnal.gov/public/hep/computing/Workshops/2012_August/index_save.shtml

[5] F. Didierjeana, G. Duchênea, A. Lopez-Martensb, The Deterministic Annealing Filter: A new clustering method for $\gamma$-ray tracking algorithms, (2010), doi:10.1016/j.nima.2010.01.030

[6] Muon (g-2) Collaboration: G.W. Bennett, et al, Final Report of the Muon E821 Anomalous Magnetic Moment Measurement at BNL, (2006), arXiv:hep-ex/0602035

[7] G. Bradski, The OpenCV Library, Dr. Dobb's Journal of Software Tools, 2236121, (2000) 